Revue de droit comparé du travail et de la sécurité sociale

3 | 2019

Les migrations internationales de travail

\title{
Le harcèlement sexuel sur le lieu de travail
}

Dominique Allen

\section{(2) OpenEdition}

Journals

Édition électronique

URL : https://journals.openedition.org/rdctss/1514

DOI : $10.4000 /$ rdctss. 1514

ISSN : 2262-9815

Éditeur

Centre de droit comparé du travail et de la sécurité sociale

Édition imprimée

Date de publication : 1 novembre 2019

Pagination : 180-183

ISSN : 2117-4350

\section{Référence électronique}

Dominique Allen, « Le harcèlement sexuel sur le lieu de travail », Revue de droit comparé du travail et de la sécurité sociale [En ligne], 3 | 2019, mis en ligne le 01 novembre 2021, consulté le 13 novembre 2021. URL : http://journals.openedition.org/rdctss/1514 ; DOI : https://doi.org/10.4000/rdctss.1514

\section{(c) $(1)(9)$}

Revue de droit comparé du travail et de la sécurité sociale est mise à disposition selon les termes de la Licence Creative Commons Attribution - Pas d'Utilisation Commerciale - Pas de Modification 4.0 International. 


\title{
DOMINIQUE ALLEN
}

\author{
UNIVERSITÉ DE MONASH
}

\section{LE HARCÈLEMENT SEXUEL SUR LE LIEU DE TRAVAIL}

Comme de nombreux pays, l'Australie a ressenti l'impact du mouvement « \#MeToo » et connaît un regain dans la lutte contre le harcèlement sexuel. En effet, le problème continue de se poser de manière persistante dans de nombreux lieux de travail australiens. Une étude nationale réalisée en 2018 par la Commission australienne des droits de l'homme (AHRC) a ainsi révélé que $23 \%$ des femmes et $16 \%$ des hommes avaient été victimes de harcèlement sexuel au travail au cours des 12 mois précédents. Le plus souvent, les victimes étaient âgées de 18 à 29 ans. Si le harcèlement sexuel existe dans tous les domaines d'activité, il est davantage susceptible de se produire dans les secteurs de l'information, des médias et des télécommunications ${ }^{1}$. Par ailleurs, il est très prégnant chez les avocats : $47 \%$ des femmes australiennes ayant participé au sondage de l'International Bar Association sur le harcèlement sexuel au sein de la profession juridique ont déclaré en avoir été victimes, une proportion supérieure de $37 \%$ par rapport au chiffre global².

Cette année, l'acteur hollywoodien Geoffrey Rush a remporté un procès en diffamation contre un journal de Sydney, Nationwide News (éditeur du Daily Telegraph), qui a été jugé coupable de diffamation pour avoir publié des articles affirmant que l'acteur s'était comporté de manière sexuellement inappropriée à l'égard d'une actrice (identifiée plus tard comme étant Eryn Jean Norvill) lorsqu'ils jouaient ensemble à Sydney dans la pièce de théâtre "Le Roi Lear ${ }^{3}$. L'affaire a mis sous les projecteurs de nombreux membres bien connus de l'industrie cinématographique australienne. Au cours du procès, Geoffrey Rush et Eryn Jean Norvill ont fait l'objet d'une grande couverture médiatique. Les deux acteurs avaient certes l'habitude de se trouver sous le feu des projecteurs, mais l'attention dont ils ont tous deux fait l'objet (à l'intérieur et à l'extérieur de la salle d'audience) fut particulièrement intense. Aussi, pour le plaignant « ordinaire », comme pour l'employeur, l'intérêt potentiel des médias vis-à-vis d'une affaire pour harcèlement sexuel constitue déjà une raison suffisante pour parvenir à un règlement en toute confidentialité.

Les clauses de confidentialité sont en effet très courantes dans les accords de règlement relatifs aux plaintes pour harcèlement sexuel. La grande majorité des plaintes pour harcèlement sexuel étant réglées à l'amiable et non devant les tribunaux, ces clauses dissimulent l'existence des plaintes pour harcèlement sexuel et cachent ainsi l'étendue du problème sur les lieux de travail en Australie.

Le harcèlement sexuel sur le lieu de travail est interdit en Australie par la législation nationale et par les lois en vigueur dans chacun des différents Etats et territoires. Toutefois,

1 Australian Human Rights Commission, «Everyone's Business: Fourth National Survey on Sexual Harassment in Australian Workplaces », 2018, p. 21: https://www.humanrights.gov.au/our-work/ sex-discrimination/public cations/everyones-business-fourth-national-survey-sexual

2 International Bar Association, "Us Too? Bullying and Sexual Harassment in the Legal Profession", 2019, p. 87 : https://www.ibanet.org/bullying-and-sexual-harassment.aspx

3 Rush v Nationwide News Pty Ltd, $n^{\circ}$ 7, 2019, FCA 496. 


\section{Australie}

les lois et leur application sont très similaires dans l'ensemble du pays. Par exemple, la loi de 1984 du Parlement australien sur la discrimination fondée sur le sexe rend illégale toute avance sexuelle non souhaitée, toute sollicitation de faveur sexuelle, ou toute autre conduite jugée importune au regard de circonstances dans lesquelles toute personne raisonnable aurait pu naturellement prévoir que le plaignant en serait offensé, humilié ou intimidé ${ }^{4}$. Un employeur sera considéré comme indirectement responsable du comportement illégal d'un salarié, à moins de pouvoir démontrer qu'il a pris toutes les mesures raisonnables pour prévenir le harcèlement sexuel ${ }^{5}$.

S'il souhaite engager des poursuites pour harcèlement sexuel, le plaignant est tenu de déposer une plainte auprès de l'AHRC fédérale ou de son agence locale pour le respect de l'égalité. Si la plainte est fondée et relève bien de la compétence de l'Agence, celleci tentera de résoudre le différend en recourant à un mode alternatif de règlement des conflits, généralement la conciliation. En dehors de l'Etat de Victoria, il est obligatoire de tenter une conciliation pour résoudre l'affaire avant que le plaignant ne porte l'affaire devant les tribunaux.

Les plaintes pour harcèlement sexuel sont les plus fréquentes parmi celles reçues par les Agences pour le respect de l'égalité. En 2017-2018, 321 plaintes ont été reçues à I'AHRC, soit $27 \%$ des plaintes enregistrées en vertu de la loi de 1984 sur la discrimination fondée sur le sexe. Les plaintes pour harcèlement sexuel figurent au $4{ }^{\mathrm{ème}}$ rang des plaintes les plus répandues parmi toutes celles déposées auprès de la Commission victorienne pour l'égalité des chances et les droits de l'homme (VEOHRC), après les plaintes pour une discrimination fondée sur le handicap ( 1 er $)$, le sexe $\left(2^{2 \mathrm{èm}}\right)$ ou l'appartenance raciale $\left(3^{\text {ème }}\right)^{6}$. Si la VEOHRC a relevé une augmentation de $20 \%$ du nombre de plaintes pour harcèlement sexuel suite au mouvement \#MeToo, l'AHRC a néanmoins constaté que seule une personne sur cinq porte officiellement plainte pour harcèlement sexuel et $20 \%$ de ceux qui l'ont fait ont été accusés d'être des fauteurs de troubles, ostracisés, victimisés ou ignorés par leurs collègues, ou ont donné leur démission.

La plupart des plaintes pour harcèlement sexuel ne sont pas portées devant les tribunaux et sont soit réglées avant d'en arriver au procès, soit retirées ${ }^{7}$. Les raisons pour lesquelles les plaignants préfèrent éviter le procès sont communément liées aux frais de justice, au temps nécessaire pour mener une action en justice, et au risque de perdre son procès. Quant aux motivations pour régler à l'amiable une plainte pour harcèlement sexuel, elles sont davantage liées au fait de devoir témoigner d'une expérience déplaisante et de subir un contre-interrogatoire, d'éviter un face-à-face avec le coupable, de tourner la page, de contourner l'intérêt des médias pour les affaires de harcèlement sexuel.

En Australie, les Agences pour l'égalité gardent confidentiels les processus qui aboutissent à la résolution des plaintes. À titre d'exemple, la loi de 1986 sur les droits de l'homme dispose que les conférences de conciliation doivent se dérouler à huis clos,

\footnotetext{
Section 28A.

Section 106.

6 Victorian Equal Opportunity and Human Rights Commission, «Rapport annuel 2017-18», p. 11.

7 Voir D. Allen, "Settling Sexual Harassment Complaints - What Benefits does ADR Offer?", Australasian Dispute Resolution Journal, 2013, vol. 24, p. 169; D. Allen, «Behind the Conciliation Doors - Settling Discrimination Complaints in Victoria», Griffith Law Review, 2009, vol. 18, $n^{\circ} 3$, p. 778.
} 
rien ne peut en filtrer ou être utilisé comme preuve si la demande est portée devant les tribunaux ${ }^{8}$. Cela permet aux parties de discuter des problèmes plus sereinement et de négocier librement.

Une telle confidentialité est essentielle au bon fonctionnement du processus de résolution des conflits. Cela signifie toutefois que les Agences hésitent à divulguer des informations concernant les plaintes pour harcèlement sexuel qu'elles reçoivent, à l'exception de leur nombre?. Ainsi, bien qu'ils soient les garants des plaintes de harcèlement sexuel, les organismes publics ne dévoilent aucune information sur la nature des plaintes reçues ou sur la manière dont elles sont résolues, y compris sur les montants des indemnisations négociées. L'étude du problème du harcèlement sexuel sur le lieu de travail n'en est que davantage compliquée. Le caractère privé du système dans son ensemble donne l'impression que la faute relève d'une affaire privée entre individus, plutôt qu>un problème social plus vaste. Pour remédier à ce manque d'informations, les organismes pourraient utilement publier sur leurs sites web et dans leurs rapports annuels des indications anonymisées sur la nature des plaintes pour harcèlement sexuel qu'ils reçoivent et sur la façon dont elles sont résolues. Malheureusement, il semble y avoir peu de motivation dans ce sens.

La confidentialité s'étend au-delà du processus de résolution des conflits à proprement parler. La plupart des accords incluent une clause de confidentialité et il est difficile de passer outre. En 2017-2018, 23 avocats ont été interrogés à Melbourne ${ }^{10}$ sur la fréquence des clauses de confidentialité dans les accords en matière de discrimination en général. Deux avocats ont déclaré que les accords amiables incluent « presque toujours » une clause de confidentialité, tandis qu'un autre a qualifié cette clause de «non négociable ». L'un des avocats a même déclaré: "Personne, à ma connaissance, n'a jamais conclu un accord qui ne soit pas confidentiel ». D'après les avocats interrogés, les employeurs utilisent la clause de confidentialité pour éviter d'ouvrir la porte aux autres victimes de harcèlement sexuel et empêcher ceux qui déposent des plaintes non fondées ou vexatoires de les poursuivre. Quant aux salariés, ils préfèrent la confidentialité dès lors qu'ils ont quitté l'entreprise, s'inquiétant des dires éventuels de leur ancien employeur à leur sujet. L'étendue de ces clauses est variable. Certaines interdisent seulement au plaignant de révéler à ses collègues de travail qu'il a porté plainte, tandis que d'autres l'empêchent de parler à quiconque des circonstances de la plainte, des parties impliquées, de la résolution du litige ou des conditions de l'accord.

8 Sections 46PK, 46PKA.

9 Pour une évaluation des contraintes imposées aux agences, voir D. Allen et A. Blackham, «Under Wraps: Secrecy, Confidentiality and the Enforcement of Equality Law in Australia and the UK», Melbourne University Law Review, 2019, vol. 43, n² 2.

10 Enquête menée par D. Allen. La recherche a été approuvée par le Comité d'éthique de la recherche humaine du ministère de la Justice à Victoria ( $\left.n^{\circ} \mathrm{CF} / 16 / 23372\right)$ et par le Comité d'éthique humaine de l'Université Monash ( $n^{\circ} 8648$ ). Les entretiens ont été menés dans le cadre d'un projet plus vaste relatif à la loi de 2010 sur l'égalité des chances. Pour une analyse complète du processus de règlement des différends prévu dans la loi, voir D. Allen, «Addressing Discrimination Through Individual Enforcement: A Case Study of Victoria », Monash Business School, Victoria, 2019 : https:// www.monash.edu/ data/assets/pdf file/0005/1898996/Addressing-Discrimination-ThroughIndividual-Enforcement D-Allen Aug-2019.pdf 


\section{AUSTRALIE}

Du point de vue de la société, la confidentialité (à la fois de l'accord et du processus de résolution des plaintes) masque l'étendue du harcèlement sexuel au travail en Australie. Dans les cas les plus extrêmes, les clauses de confidentialité ont un effet dissuasif sur les plaignants qui craignent des répercussions s'ils évoquaient un aspect particulier de leur affaire. Dès lors, ils protègent leurs agresseurs, tant sur leur lieu de travail actuel que dans tous les endroits où ils pourront travailler à l'avenir.

En 2018, Kate Jenkins, la Commissaire fédérale chargée de la discrimination sexuelle, a lancé une enquête nationale sur le harcèlement sexuel au travail en Australie. Reconnaissant que les clauses de confidentialité étaient de nature à interdire aux personnes ayant conclu des accords après des plaintes pour harcèlement sexuel de témoigner dans le cadre cette enquête, la Commissaire a invité les employeurs à lever partiellement les obligations de confidentialité, de manière à ce que les personnes concernées puissent soumettre un témoignage confidentiel et ainsi mieux l'aider à comprendre les causes sous-jacentes du harcèlement sexuel sur le lieu de travail et les situations susceptibles de le provoquer ${ }^{11}$. Cependant, 38 employeurs seulement ont accepté cette dérogation. Ils sont cités dans une liste publiée sur le site de l'AHRC et comptent de grandes entreprises, des universités et des organismes du secteur public. Au grand regret de Kate Jenkins, de nombreuses entreprises internationales ne figurent pas sur cette liste ${ }^{12}$.

Le rapport de l'enquête devrait être publié au plus tard d'ici la fin de l'année. Il faut espérer qu'il permettra de mieux cerner la recrudescence de ces clauses de confidentialité, leur fonctionnement et leur impact - en bien ou en mal - sur les salariés comme les employeurs, et d'envisager de nouvelles formulations permettant de protéger les intérêts des parties, sans occulter la prévalence du harcèlement sexuel sur le lieu de travail. Le mouvement \#MeToo constitue en cela un rappel du fait que le harcèlement sexuel existe toujours et ne pourra être éradiqué que si nous connaissons les raisons originelles du problème et sa véritable ampleur.

11 Voir la lettre de novembre 2018, écrite par Kate Jenkins, Commissaire chargée de la discrimination sexuelle: https://www.humanrights.gov.au/national-inquiry-sexual-harassment-australian-workplaceslimited-waiver-confidentiality-obligations

12 E. Steel, «Employers who talk up gender equity but silence harassment victims » (Employeurs qui parlent d'égalité des sexes mais font taire les victimes de harcèlement), The New York Times, $1^{\mathrm{er}}$ mai 2019. 